\title{
ORAL N-ACETYLCYSTEINE LOWERS PLASMA HOMOCYSTEINE IN ADULTS ON A BACKGROUND OF ANABOLIC RESISTANCE TRAINING
}

\author{
W. Hildebrandt ${ }^{1,2}$, H. Krakowski-Roosen ${ }^{2,3}$, H. Renk ${ }^{2,4}$, A. Künkele ${ }^{2,5}$, R. Sauer ${ }^{2,6}$, D. Tichy ${ }^{7}$, \\ L. Edler ${ }^{7}$, R. Kinscherf ${ }^{1}$
}

\begin{abstract}
Lowering high plasma levels of homocysteine (tHcy) by folate/vitamin-B-supplementation only unsufficiently protects against cardiovascular diseases and dementia. To enhance therapeutic options, we evaluated whether the significant tHcy-lowering effect of oral N-acetylcysteine (NAC) in sedentary adults $(-11.71 \%$ [12]) is still detectable on a background of anabolic resistance training (RT) which moderately decreases tHcy itself. Reanalysing a previous randomized controlled double-blinded clinical trial, we compared the effect of oral NAC ( 8 weeks $1.8 \mathrm{~g} / \mathrm{d}, \mathrm{n}=9)$ to that of placebo $(\mathrm{n}=8)$ on postabsorptive tHcy in healthy middleaged subjects (tHcy $11.82 \pm 0.69 \mu \mathrm{M}$ ) undergoing 8 weeks of supervised progressive RT. NAC (+RT) led to a significantly greater reduction of tHcy $(-13.97 \pm 5.81 \%)$ than placebo $(+\mathrm{RT})(-3.85 \pm 4.81 \%)$ as confirmed by ANOVA $(\mathrm{P}<0.05)$ adjusting for methionine plasma levels and gain in strength. This add-on effect of NAC $(\sim-10 \%)$ suggests that combining cysteine supplementation with RT may offer a novel (additional) option to lower tHcy in an aging population.
\end{abstract}

Key words: Aging, exercise, thiol, cysteine, prevention.

\section{Introduction}

Elevated total plasma levels of homocysteine (tHcy) has long been considered to be a pro- oxidative/inflammatory risk factor of endothelial dysfunction, atherosclerosis and related cardiovascular endpoints (1-3). However, available large-scale trials on tHcy-lowering $(\sim-25 \%)$ through folate/B-vitamins supplementation have shown the cardiovascular benefit to be limited to stroke (1). Presently, in line with its agerelated increase and its role in oxidative stress (4), tHcy is emerging as a factor of age-related neuronal degeneration though the benefit of folate/ B-vitamins remains to be proven (5). Moreover, tHcy is implicated in the age-

1. Department of Medical Cell Biology, Institute of Anatomy and Cell Biology, Philipps-University of Marburg, Robert-Koch-Str. 8, 35032 Marburg, Germany; 2. Former Department of Immunochemistry, Deutsches Krebsforschungszentrum, Im Neuenheimer Feld 280, D-69120 Heidelberg, Germany; 3. Applied Sport Sciences, University of Applied Sciences Hamm-Lippstadt, Marker Allee 76-78, 59063 Hamm, Germany; 4. University Children's Hospital Tübingen, Department of Paediatric Cardiology, Pulmology and Intensive Care Medicine, Hoppe-Seyler Str. 1, 72076 Tübingen, Germany; 5. Department of Pediatric Oncology and Hematology, Charité - University Hospital Berlin, Augustenburger Platz 1, 13353 Berlin, Germany; 6. Department of Neurology, General Hospital Fürth, JakobHenle-Straße 1, 90766 Fürth, Germany; 7. Division of Biostatistics, Deutsches Krebsforschungszentrum, Im Neuenheimer Feld 280, 69120 Heidelberg, Germany

Corresponding Author: Prof. Dr. med. Wulf Hildebrandt, Department of Medical Cell Biology, Institute for Anatomy and Cell Biology, University of Marburg, Robert-Koch-Straße 8, 35032 Marburg, Tel. +49-6421-28-64042, Fax: +49-6421-28 -68983, e-mail: Wulf.Hildebrandt@staff.uni-marburg.de related decline of skeletal muscle mass and function which critically limit mobility and life-span (6-8).

Given the insufficient prevention through folate/Bvitamins, alternative/additional options for tHcylowering (ideally via different mechanisms) are needed: Resistance training (RT), strongly suggested for maintenance of skeletal muscle mass (8), has been shown to moderately lower tHcy by 5-6\% possibly via methionine incorporation into myofibrillar proteins (9, 10).

As another option, the thiol compound $\mathrm{N}$-acetylcysteine (NAC) is considered to lower tHcy by increasing renal tHcy clearance via thiol-exchange at (albumin) disulfide-binding sites $(11,12)$. Upon intravenous bolus application, NAC acutely lowers tHcy by up to $-50 \%$ (2). More relevant to primary prevention, several weeks of oral $1.8 \mathrm{~g} / \mathrm{d}$ NAC lead to a tHcy decrease by $-11.7 \%$ which is associated with reductions in blood pressure (12). While this NAC effect on tHcy was demonstrated in healthy sedentary adults, it remains to be proven on a background of RT, because RT lowers tHcy itself.

We therefore explored unpublished data of a randomized, double-blind, placebo-controlled trial on the effect of 8 weeks $1.8 \mathrm{~g} / \mathrm{d}$ NAC orally taken during an 8-weeks-program of anabolic RT in healthy adults. The tHcy reduction attributable to NAC was quantified 
and compared with the outcome of a previous trial on an identical dose of oral NAC dose in sedentary subjects (12).

\section{Methods}

\section{Subjects}

Seventeen healthy normotensive middle-aged adults were recruited to the randomized placebo-controlled trial on a background of progressive RT (Table 1). Calculation of sample size ( $n=8-9$ per treatment arm) was based on published tHcy-lowering effects of NAC (12) or RT (10) alone. The study was approved by the Ethical Committee the University of Heidelberg (L-157/2003-2, 11.11.2003) and complied with the Declaration of Helsinki (1996). No registration in an ICMJE-approved public trials registries had been required for this study completed before June 2004. Main exclusion criteria were: tHcy $>30$ $\mu \mathrm{M}, \mathrm{NAC}$ or vitamin supplementation, NAC intolerance, cardiovascular, renal, metabolic or any other disease. Body composition was analysed by measurement of electrical impedance and reactance using the TVI-10 body composition analyzer (FM Service $\mathrm{GmbH}$, Leverkusen, Germany).

\section{Trial medication and supplementation}

1.8 g per day NAC (Fluimucil, Zambon, Bresso, Italy) or placebo (Lactose) were taken orally over 8 weeks as 3x3 $200 \mathrm{mg}$ capsules (white, size 2, blinded with regard to the characteristic NAC smell). To exclude nutritional or endogeneous limitations in creatine availability during RT (10), $1 \mathrm{~g} / \mathrm{d}$ oral creatine (DSM Fine Chemicals Austria $\mathrm{GmbH}$, Linz, Austria) was supplemented throughout.

\section{Blood parameters}

Antecubital venous blood samples were drawn between 8:00 and 10:00 a.m. after $\geq 12 \mathrm{~h}$ overnight fast and $>48 \mathrm{~h}$ abstinence from RT for fluormetric determination of tHcy by high-performance-liquid-chromatography (HPLC; Abbott Laboratory, Wiesbaden, Germany). The acid-soluble plasma thiol concentration was measured photometrically and the acid-soluble plasma concentrations of cystine (cysteine-disulfide) and of methionine determined by HPLC (Amino Acid Analyzer LC 3000, Eppendorf, Hamburg, Germany) as described $(12,13)$.

\section{Training intervention}

The 8-week-protocol of progressive concentric isokinetic RT of the knee extensors and flexors comprised 16 professionally supervised sessions (60 $\mathrm{min}, 2 /$ week) using the Multi-Joint-System Isomed-2000 ${ }^{\mathrm{TM}}$ (D+R Ferstl, Hemau, Germany). The subjects' knee extensor peak torque $(\mathrm{PT})$ was assessed under isokinetic $\left(80^{\circ}\right.$ range of motion (ROM), angular velocity (AV) $60^{\circ} \mathrm{s}^{-1}$ ) and isometric (flexion angle of $40^{\circ}, 3$ maximal voluntary contractions covering $7 \mathrm{~s}$ ) conditions at each session. The isokinetic training consisted of three sets of 12 flexionextension-cycles at (progressively adjusted) $75 \%$ of the individual isokinetic PT at a ROM of $80^{\circ}$ and $\mathrm{AV}$ of $60^{\circ} \mathrm{s}^{-1}$, was performed by the left and the right leg separately and guided by visual monitor feed-back of a preset individual torque trace.

\section{Statistics}

Descriptive statistical analyses report means ( \pm standard error of the mean (S.E.M.)) of the quantitative characteristics collected on pre- and post-treatment as well as their intra-individual absolute and percentage changes (Table 1). Differences between the two arms, i.e. $\mathrm{NAC}+\mathrm{RT}$ and placebo+RT, were tested for tHcy as the primary endpoint as well as for the secondary endpoints by the Student's two-sample unpaired t-test (Table 1). Differences in tHcy changes between the NAC+RT and placebo+RT arms were also assessed using an analysis of variance (ANOVA) to adjust for covariate effects, in particular i) the plasma level of methionine as a major source of tHcy and ii) the RT-related gain in isometric PT. Furthermore, a multivariate analysis of variance (MANOVA) was applied to test for the interaction 'time' by 'medication' (NAC versus placebo) as described [12]. In addition, the paired t-test or the Wilcoxon test when the t-test was inadequate was applied to each treatment arm, to detect significant differences between pre-and post-treatment values. P-values were reported as statistically significant when $\mathrm{P}<0.05$. The SPSS-software (version 22.0 SPSS Inc., Chicago, IL, USA) was used throughout.

\section{Results}

Baseline anthropometric data, muscle function, as well as plasma amino acids were comparable between the NAC and placebo treatment arms (Table 1). Mean baseline tHcy of the total study population was $11.83 \pm 0.70$ $\mu \mathrm{M}$, i.e. slightly above the values $(9.53 \pm 0.35 \mu \mathrm{M})$ of our previous trial on NAC in 82 sedentary subjects [12] which had been ca 8 years younger ( $43.5 \pm 3.5$ vs. $51.7 \pm 2.1$ years). Eight weeks of RT yielded substantial and significant increases in isometric and isokinetic PT in both treatment arms, differing neither in absolute nor in percentage terms of strength gain (Table 1). The concomitant small increases in body weight, BMI and BCM (at stable body fat) were also not different between the NAC and placebo arm (though significant within the NAC arm). As a main finding, tHcy significantly decreased with 8 weeks of NAC treatment $(-13.97 \pm 5.81 \%, \mathrm{p}=0.046$ by paired t-test) but not with placebo $(-3.85 \pm 4.81 \%)$ (Table 1; Fig. 1, right panel). For comparison, in our previous 
Table 1

Anthropometry, muscle function and amino acid plasma levels before and after NAC and placebo treatment during ongoing resistance training

\begin{tabular}{|c|c|c|c|c|c|c|}
\hline & & Treatment arm & Pre-treatment & Post-treatment & $\Delta$ absolute & $\Delta \%$ \\
\hline \multicolumn{7}{|c|}{ Anthropometry, muscle function } \\
\hline \multirow[t]{2}{*}{$\mathrm{N}$} & & Placebo & $8(6 \mathrm{f} / 2 \mathrm{~m})$ & 8 & & \\
\hline & & NAC & $9(4 \mathrm{f} / 5 \mathrm{~m})$ & 9 & & \\
\hline \multirow[t]{2}{*}{ Age } & (years) & Placebo & $53.6 \pm 3.1$ & & & \\
\hline & & NAC & $50.0 \pm 3.0$ & & & \\
\hline \multirow[t]{2}{*}{ Body height } & $(\mathrm{cm})$ & Placebo & $171.1 \pm 2.8$ & & & \\
\hline & & NAC & $170.3 \pm 3.6$ & & & \\
\hline \multirow[t]{2}{*}{ Body weight } & $(\mathrm{kg})$ & Placebo & $73.5 \pm 7.0$ & $73.8 \pm 7.2$ & $0.28 \pm 0.37$ & $0.25 \pm 0.54$ \\
\hline & & NAC & $70.1 \pm 5.6$ & $71.6 \pm 5.7^{*}$ & $1.50 \pm 0.48$ & $2.19 \pm 0.71$ \\
\hline \multirow[t]{2}{*}{ BMI } & $\left(\mathrm{kg} \mathrm{m}^{2}\right)$ & Placebo & $25.42 \pm 1.82$ & $25.58 \pm 1.83$ & $0.07 \pm 0.13$ & $0.24 \pm 0.54$ \\
\hline & & NAC & $23.73 \pm 1.26$ & $24.23 \pm 1.25$ * & $0.50 \pm 0.17$ & $2.19 \pm 0.71$ \\
\hline \multirow[t]{2}{*}{$\mathrm{BCM}$} & $(\mathrm{kg})$ & Placebo & $32.49 \pm 3.25$ & $33.07 \pm 3.79$ & $0.59 \pm 1.03$ & $1.23 \pm 3.54$ \\
\hline & & NAC & $29.84 \pm 2.45$ & $31.18 \pm 2.58$ ** & $1.33 \pm 0.34$ & $4.45 \pm 0.99$ \\
\hline \multirow[t]{2}{*}{ Body fat } & $(\%)$ & Placebo & $21.11 \pm 2.43$ & $21.50 \pm 2.46$ & $0.39 \pm 1.30$ & $4.10 \pm 7.64$ \\
\hline & & NAC & $20.66 \pm 1.49$ & $21.28 \pm 2.08$ & $0.62 \pm 1.32$ & $2.65 \pm 6.43$ \\
\hline \multirow[t]{2}{*}{ Isometric PT } & $(\mathrm{Nm})$ & Placebo & $155.0 \pm 13.0$ & $199.1 \pm 15.6$ ** & $44.04 \pm 10.93$ & $30.61 \pm 9.63$ \\
\hline & & NAC & $153.2 \pm 15.0$ & $185.8 \pm 13.3^{* * *}$ & $32.60 \pm 4.53$ & $24.82 \pm 5.63$ \\
\hline \multirow[t]{2}{*}{ Isokinetic PT } & $(\mathrm{Nm})$ & Placebo & $160.6 \pm 17.4$ & $183.2 \pm 22.9$ * & $22.61 \pm 7.16$ & $12.97 \pm 3.52$ \\
\hline & & NAC & $162.9 \pm 16.3$ & $187.3 \pm 17.7$ * & $24.38 \pm 7.94$ & $16.04 \pm 5.29$ \\
\hline \multicolumn{7}{|l|}{ Plasma amino acid levels } \\
\hline \multirow[t]{2}{*}{ Homocysteine (tHcy) } & $(\mu \mathrm{M})$ & Placebo & $11.18 \pm 0.71$ & $10.62 \pm 0.57$ & $-0.56 \pm 0.52$ & $-3.85 \pm 4.81$ \\
\hline & & NAC & $12.40 \pm 1.15$ & $10.38 \pm 0.80$ * & $-2.02 \pm 0.86 \S$ & $-13.97 \pm 5.81$ \\
\hline \multirow[t]{2}{*}{ Thiol } & $(\mu \mathrm{M})$ & Placebo & $5.23 \pm 0.32$ & $5.47 \pm 0.47$ & $0.24 \pm 0.52$ & $6.41 \pm 10.73$ \\
\hline & & NAC & $5.29 \pm 0.42$ & $6.27 \pm 0.78$ & $0.95 \pm 1.13$ & $34.73 \pm 30.71$ \\
\hline \multirow[t]{2}{*}{ Cystine } & $(\mu \mathrm{M})$ & Placebo & $36.28 \pm 1.96$ & $34.19 \pm 2.89$ & $-2.1 \pm 1.28$ & $-6.41 \pm 3.44$ \\
\hline & & NAC & $34.57 \pm 1.32$ & $36.22 \pm 1.86$ & $1.66 \pm 1.70$ & $5.21 \pm 4.92$ \\
\hline \multirow[t]{2}{*}{ Methionine } & $(\mu \mathrm{M})$ & Placebo & $19.28 \pm 2.87$ & $19.40 \pm 3.24$ & $0.17 \pm 0.70$ & $1.88 \pm 3.61$ \\
\hline & & NAC & $16.79 \pm 2.28$ & $18.87 \pm 2.63$ * & $2.08 \pm 0.73$ & $13.74 \pm 6.32$ \\
\hline
\end{tabular}

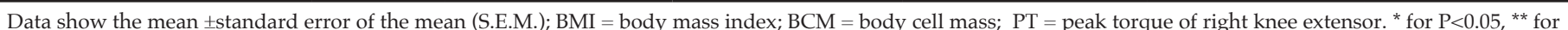

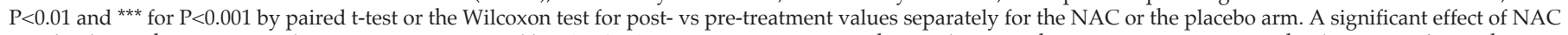

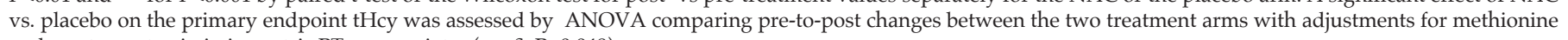
and pre-to-post gain in isometric PT as covariates (see $\S, \mathrm{P}=0.048$ ).

placebo-controlled trial in sedentary male subjects (Fig. 1, left panel,[12]) tHcy significantly decreased with NAC $(-11.71 \pm 3.04 \%, \mathrm{P}<0.001)$ but not with placebo $(4.09 \pm 3.59 \%$, $\mathrm{P}>0.05)$. ANOVA with adjustment for plasma methionine levels and gain in isometric PT detected a significant difference between the NAC and placebo effect on tHcy $(\mathrm{P}=0.048$; see $\S$ in Fig. 1 and Table 1$)$. This result was further scrutinized and confirmed by MANOVA $(\mathrm{P}=0.048$, factor 'time' by 'medication') when adjusting for the same covariates. The increase in plasma thiol was found to be non-significantly higher with NAC $(0.95 \pm 1.13$ $\mu \mathrm{M}, 34.73 \pm 30.71 \%)$ than with placebo $(0.24 \pm 0.52 \mu \mathrm{M}$, $6.41 \pm 10.73 \%$ ). A similar trend was observed for plasma cystine (cysteine-disulfide). Methionine was significantly increased with NAC only $(\mathrm{P}=0.02)$ (Table 1$)$. 
Figure 1

Total homocysteine plasma levels (tHcy) before and after $1.8 \mathrm{~g} / \mathrm{d}$ oral NAC or placebo treatment of non-exercising subjects (previous study (12), left panel,n=82) and of subjects undergoing anabolic resistance training (present study, right panel, $\mathrm{n}=17$ )

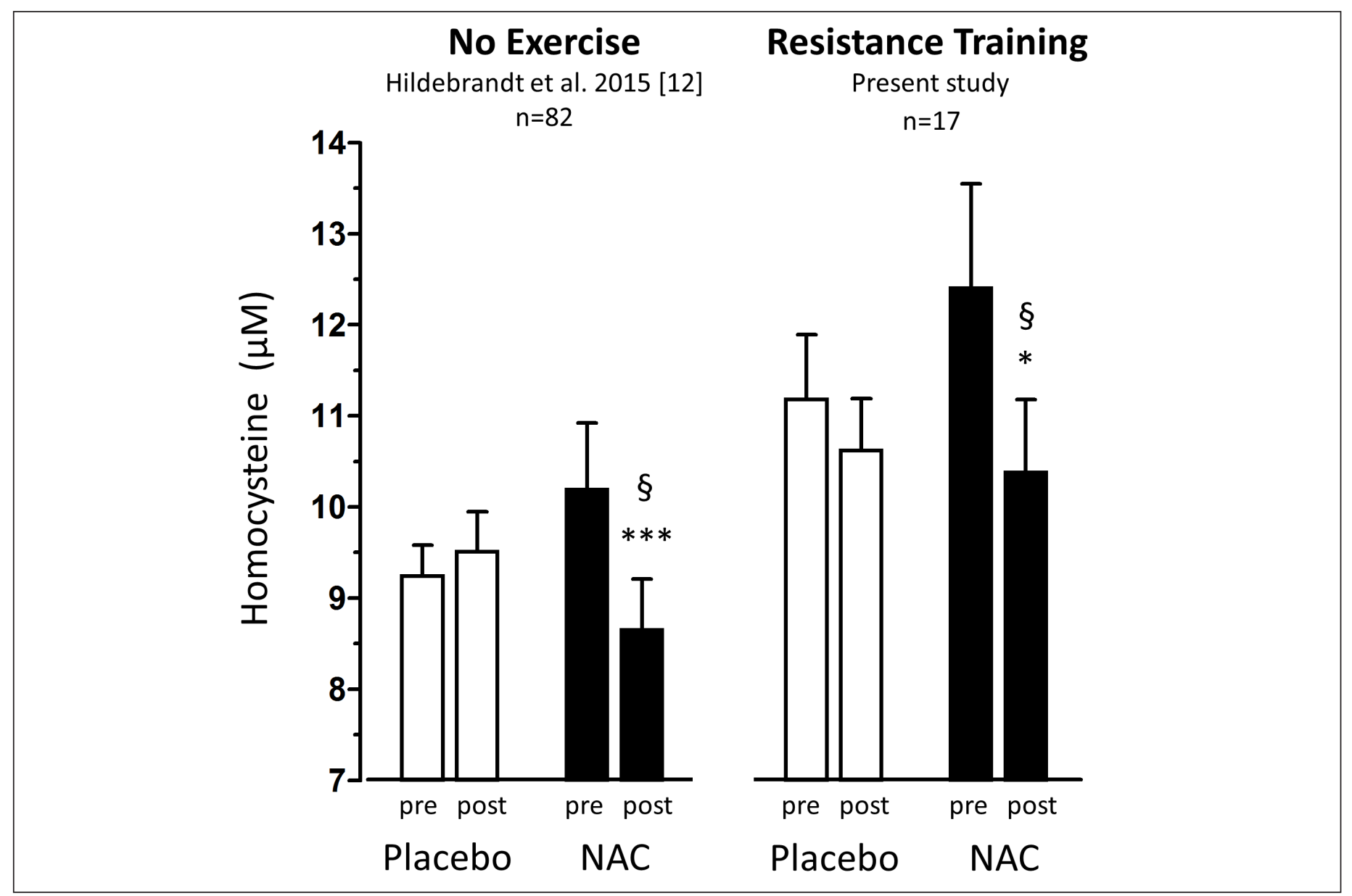

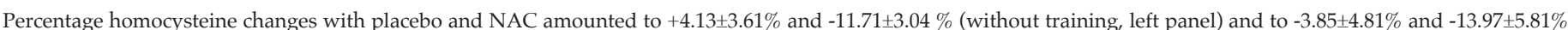

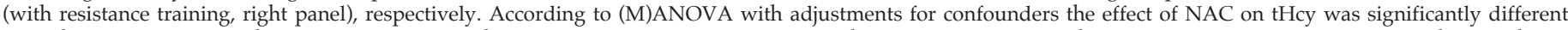

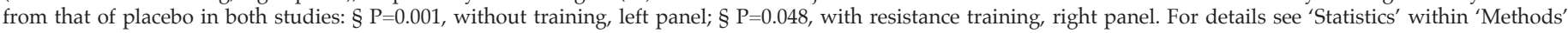
section. Data represent means \pm S.E.M.; * for $\mathrm{p}>0.05$ and ${ }^{* *}$ for $\mathrm{p}<0.001$ by Student's t-test for paired observation.

\section{Discussion}

Though of limited size, this randomized double-blind clinical trial showed for the first time (generated the hypothesis), that a dose of $1.8 \mathrm{~g}$ NAC / d for 8 weeks, orally taken on a background of effectively anabolic RT, significantly lowered tHcy by $-13.97 \%$, while a nonsignificant decrease of $-3.85 \%$ tHcy occured with placebo (RT alone). This resulting 'add-on' effect of NAC of around $-10 \%$ tHcy reduction is well in line with our recent findings in non-training males $(-11.71 \pm 3.04 \%)$ taking an identical oral NAC dose (12). Notably, two covariates were presently identified to significantly impact the detected NAC effect on tHcy (warranting consideration as confounders in previous and future trials): The RT-related (likely anabolic) gain in isometric $\mathrm{PT}$ and the plasma methionine level as a main and nutritionally variable source of tHcy (3). Likely due to the limited muscle mass involved (lower limb vs. whole body) the presently observed tHcy-lowering of $-3.85 \%$ with RT (plus placebo) remained slightly below the published range of -5 to $-6 \%(9,10)$ despite creatine supplementation. Importantly however, NAC did not compromise the outcome of RT suggested as an antiaging intervention. Of note, the present postabsorptive measurements ( $12 \mathrm{~h}$ after the last NAC dose) may not reflect the transient NAC-induced increase in plasma thiol (mainly cysteine) levels (13) which likely is associated with a large transient tHcy decrease of up to $-45 \%(2,11)$. The potential of NAC to acutely decrease tHcy - largely underestimated under postabsorptive conditions - might offer an option to attenuate predictable diurnal/nutritional tHcy peaks by well-timed and adjusted NAC intake.

As NAC was tolerated well and without adverse effect on the outcome of RT, a combination of oral 
NAC with RT warrants further evaluation as an antiaging intervention against tHcy-related degeneration limiting functional capacity. Indeed, in elderly subjects (>75 years), the combination of $1.8 \mathrm{~g} / \mathrm{d}$ NAC with RT was previously shown by us to significantly enhance functional capacity while decreasing plasma TNF $\alpha$ levels (14). Moreover, NAC is able to improve both, the ventilatory and the erythropoietin response to hypoxia (13) beside several other vital functions which clearly decline with age and respond to thiol redox signals - in line with a non-radical oxidative stress theory of aging (15).

Acknowledgments: We gratefully acknowledge the expert laboratory assistance of Ute Winter and Helge Lips.

Conflict of Interest: The authors declare that they have no conflict of interest.

Ethical Standards: The authors declare that the experiment (clinical trial) complied with the current law of the country (Germany) where they were performed. The study was approved approved by the Ethical Committee the University of Heidelberg (L-157/2003-2, 11.11.2003).

\section{References}

1. Martí-Carvajal AJ, Solà I, Lathyris D. Homocysteine-lowering interventions for preventing cardiovascular events. Cochrane Database Syst Rev. 2017 Aug 17;8:CD006612.doi: 10. 1002/14651858.CD006612.put;

2. Scholze A, Rinder C, Beige J, Riezler R, Zidek W, Tepel M. Acetylcysteine reduces plasma homocysteine concentration and improves pulse pressure and endothelial function in patients with end-stage renal failure. Circulation 2004;109:369-374.

3. Kanani PM, Sinkey CA, Browning RL, Allaman M, Knapp HR, Haynes WG. Role of oxidant stress in endothelial dysfunction produced by experimental hyperhomocys-t(e)inemia in humans. Circulation 1999;100:1161-1168.
4. Ventura E, Durant R, Jaussent A, Picot MC, Morena M, Badiou S, et al Homocysteine and inflammation as main determinants of oxidative stress in the elderly. Free Radic Biol Med 2009;46:737-744.

5. Clarke R, Bennett D, Parish S, Lewington S, Skeaff M, Eussen SJ, et al. B-Vitamin Treatment Trialists' Collaboration. Effects of homocysteine lowering with B vitamins on cognitive aging: meta-analysis of 11 trials with cognitive data on 22,000 individuals. Am J Clin Nutr 2014;100:657-666. doi 10.3945/ajcn.113.076349. Epub 2014 Jun 25.

6. Veeranki S, Winchester LJ, Tyagi SC. Hyperhomocysteinemia associated skeletal muscle weakness involves mitochondrial dysfunction and epigenetic modifications. Biochim Biophys Acta 2015;1852:732-741. doi: 10.1016/j. bbadis.2015.01.008. Epub 2015 Jan 20.

7. Guralnik JM, Ferrucci L, Simonsick EM, Salive ME, Wallace RB. Lowerextremity function in persons over the age of 70 years as a predictor of subsequent disability. N Engl J Med 1995;332:556-561.

8. Liu CJ, Latham NK. Progressive resistance strength training for improving physical function in older adults. Cochrane Database Syst Rev. 2009; 8(3):CD002759. doi: 10.1002/14651858.CD002759.

9. Silva Ade S, da Mota MP. Effects of physical activity and training programs on plasma homocysteine levels: a systematic review. Amino Acids 2014;46:1795-1804.

10. Steenge GR, Verhoef P, Greenhaff PL. The effect of creatine and resistance training on plasma homocysteine concentration in healthy volunteers. Arch Intern Med 2001;161:1455-1456.

11. Ventura P, Panini R, Abbati G, Marchetti G, Savioli G. Urinary and plasma homocysteine and cysteine levels during prolonged oral $\mathrm{N}$-acetylcysteine therapy. Pharmacology 2003; 68:105-114.

12. Hildebrandt W, Sauer R, Bonaterra G, Dugi KA, Edler L, Kinscherf R. Oral $\mathrm{N}$-acetylcysteine reduces plasma homocysteine concentrations regardless of lipid or smoking status. Am J Clin Nutr 2015;102:1014-1024.

13. Hildebrandt W, Alexander S, Bartsch P, Dröge W. Effect of N-acetylcysteine on the hypoxic ventilatory response and erythropoietin production linkage between plasma thiol redox state and O2-Chemosensitivity. Blood 2002;99:1552-1555.

14. Hauer K, Hildebrandt W, Sehl Y, Edler L, Oster P, Dröge W. Improvement in muscular performance and decrease in tumor necrosis factor level in old age after antioxidant treatment. J Mol Med 2003; 81:118-125.

15. Go YM, Jones DP. Redox theory of aging: implications for health and disease Clin Sci (Lond) 2017; 31:1669-1688. doi: 10.1042/CS20160897. Print 2017 Jul 15. 